CERN-TH.6970/93

hep-th/9308042

(revised)

\title{
On field redefinitions and exact solutions in string theory
}

\author{
A.A. Tseytlin* \\ Theory Division, CERN \\ CH-1211 Geneva 23, Switzerland \\ and \\ Theoretical Physics Group, Blackett Laboratory \\ Imperial College \\ London SWr 2BZ, U.K.
}

\begin{abstract}
String backgrounds associated with gauged $G / H$ WZNW models in general depend nontrivially on $\alpha^{\prime}$. We note, however, that there exists a local covariant $\alpha^{\prime}$-dependent field redefinition that relates the exact metric-dilaton background corresponding to the $S L(2, R) / U(1)$ model to its leading-order form $(D=2$ black hole). As a consequence, there exists a 'scheme' in which the string effective equations have the latter as an exact solution. However, the corresponding equation for the tachyon (which, like other Weyl anomaly equations, has scheme-dependent form) still contains corrections of all orders in $\alpha^{\prime}$. As a result, the string 'probes' still feel the $\alpha^{\prime}$-corrected background. The field redefinitions we discuss contain the dilaton terms in the metric transformation law. We also comment on exact forms of the duality transformation in different 'schemes'.
\end{abstract}

CERN-TH.6970/93

August 1993

* On leave from Lebedev Physics Institute, Moscow, Russia. e-mail: tseytlin@surya3.cern.ch and tseytlin@ic.ac.uk 


\section{General remarks on field redefinition ambiguity}

String theory is effectively a non-local theory when represented in terms of the standard local fields. If ones uses the couplings $\varphi^{i}=\left(G_{\mu \nu}, B_{\mu \nu}, \phi, \ldots\right)$ of a $2 d \sigma$-model to parametrise string backgrounds then the (string tree level) effective action $S\left(\varphi^{i}\right)$ [1] or the corresponding equations ( $\sigma$-model conformal invariance conditions $\left.\bar{\beta}^{i}=0\right)$ contain terms of all orders in derivatives of the fields multiplied by powers of $\alpha^{\prime}$,

$$
\bar{\beta}^{i}=\kappa^{i j} \frac{\partial S}{\partial \varphi^{j}}=\bar{\beta}_{1}^{i}(\varphi)+\alpha^{\prime} \bar{\beta}_{2}^{i}(\varphi)+\ldots=0
$$

Since $\bar{\beta}_{1}^{i}$ are of second order in derivatives, solving (1) perturbatively in $\alpha^{\prime}$ we find

$$
\varphi_{*}^{i}=\varphi_{1}^{i}+\alpha^{\prime} \varphi_{2}^{i}+\ldots, \quad \varphi_{2}^{i}=-\left[\left(\frac{\partial \bar{\beta}_{1}^{i}}{\partial \varphi^{j}}\right)^{-1} \bar{\beta}_{2}^{j}\right]\left(\varphi_{1}\right), \ldots
$$

where higher-order corrections $\varphi_{2}^{i}, \varphi_{3}^{i}, \ldots$, are in general non-local, being expressed in terms of the leading-order solution $\varphi_{1}^{i}$.

If the only information one uses is an on-shell string $S$-matrix, then the effective action is defined modulo local field redefinitions (since they do not change the $S$-matrix) [2] [3]

$$
\varphi^{i \prime}=\varphi^{i}+\alpha^{\prime} T_{1}^{i}(\varphi)+\ldots
$$

Here $T_{n}^{i}$ are local combinations of the fields containing $2 n$ derivatives. A similar ambiguity (or 'scheme dependence') is present in the $\bar{\beta}^{i}$-functions. preserve the structure of $S$ and $\bar{\beta}^{i}$, i.e. should respect their symmetries (like general covariance and $\phi \rightarrow \phi+$ const). The transformation (3) changes the structure of higherorder terms in $S$ and in $\bar{\beta}^{i}$, e.g.

$$
\bar{\beta}_{2}^{i \prime}=\bar{\beta}_{2}^{i}+\frac{\partial \bar{\beta}_{1}^{i}}{\partial \varphi^{j}} T_{1}^{j}, \quad \ldots
$$

1 This is not exactly the same as the scheme ambiguity present in the standard $\beta$-functions; the redefinitions we consider are of more general type, see below. 
and, as a result, of the solution (2). The solution of the transformed equations will, in general, remain a non-local functional of $\varphi_{1}^{i}$.

It may happen that there exists such a special representative (or a 'special scheme') in the class of equivalent (redefinition-related) actions $S$ or conformal anomaly coefficients $\bar{\beta}^{i}$ for which all higher-order corrections in (1) vanish on a particular leading-order solution $\varphi_{1}^{i}$ (so that this leading-order solution is actually an exact one). Then the solution for a generic choice of $S$ (or in a generic 'scheme') will be a function of $\alpha$ ' given by a local redefinition (3) of the special scheme solution $\varphi_{1}^{i}$. A well-known example is provided by the parallelizable spaces [4] (in particular, by group spaces corresponding to WZNW theories [5]) : there exists a special scheme [6] [7] in which each term $\bar{\beta}_{n}^{i}$ in (1) vanishes, being evaluated on $\varphi_{*}^{i}=\varphi_{1}^{i}$. The solution in a general scheme will be a 'deformation' of $G_{* \mu \nu}$ and $B_{* \mu \nu}$ by local $\alpha^{\prime}$-dependent terms (i.e. by $a_{1} \alpha^{\prime} R_{* \mu \nu}+\ldots$ and $a_{2} \alpha^{\prime} D_{*}^{\lambda} H_{* \lambda \mu \nu}+\ldots$ ). 2

In the previous example the dilaton is constant in the special scheme and remains constant in all other schemes as well. The situation becomes more subtle once the dilaton is non-constant for a leading-order solution. This is the case that we will be interested in below. We shall ignore all other fields in the theory, except the metric and the dilaton. There exists a scheme [9] [7] in which there is only one $\left(R_{\lambda \mu \nu \sigma}^{2}\right) \alpha^{2}$-term in the effective action

$$
S=\int d^{D} x \sqrt{G} \mathrm{e}^{-2 \phi}\left\{\frac{1}{6}(D-C)-\frac{1}{4} \alpha^{\prime}\left[R+4\left(\partial_{\mu} \phi\right)^{2}\right]-\frac{1}{16} \alpha^{\prime 2} R_{\mu \nu \lambda \kappa}^{2}+O\left(\alpha^{\prime 3}\right)\right\}
$$

where $C$ is a total central charge. The corresponding equations are linear combinations of the $\bar{\beta}^{i}$-functions

$$
\bar{\beta}_{\mu \nu}^{G}=R_{\mu \nu}+2 D_{\mu} D_{\nu} \phi+\frac{1}{2} \alpha^{\prime} R_{\mu \lambda \rho \sigma} R_{\nu}^{\lambda \rho \sigma}+O\left(\alpha^{\prime 2}\right)=0
$$

2 For group spaces this amounts to a rescaling of $G_{\mu \nu}$ and $B_{\mu \nu}$ by functions of $\alpha^{\prime} R\left(=\frac{1}{2} D c_{G} / k\right)$. This changes the distribution of contributions to the central charge $\| \mathbb{8} C=D k /\left(k+\frac{1}{2} c_{G}\right)$ coming from different loop orders in $\bar{\beta}^{\phi}$. In particular, one can find a scheme in which $G_{\mu \nu}$ is effectively rescaled by $k /\left(k+\frac{1}{2} c_{G}\right.$ ) (which amounts to changing $\alpha^{\prime}$ from $1 / k$ to $1 /\left(k+\frac{1}{2} c_{G}\right)$ ) so that the whole central charge comes only from the 'one-loop' $O\left(\alpha^{\prime}\right)$ correction to $\bar{\beta}^{\phi}$. 


$$
\bar{\beta}^{\phi}=\frac{1}{6}(D-C)+\alpha^{\prime}\left[-\frac{1}{2} D^{2} \phi+(\partial \phi)^{2}\right]+\frac{1}{16} \alpha^{\prime 2} R_{\lambda \mu \nu \rho}^{2}+O\left(\alpha^{\prime 3}\right)=0 .
$$

In a general scheme the $\alpha^{\prime}$-terms in (6) and $\alpha^{\prime 2}$-terms in (5),(7) will depend on a number of free parameters, which enter the most general local field redefinition [2] [7] $]^{3}$

$$
\begin{gathered}
G_{\mu \nu}^{\prime}=G_{\mu \nu}+\alpha^{\prime}\left[d_{1} R_{\mu \nu}+d_{2} \partial_{\mu} \phi \partial_{\nu} \phi+G_{\mu \nu}\left(d_{3} R+d_{4} D^{2} \phi+d_{5}(\partial \phi)^{2}\right)\right]+O\left(\alpha^{\prime 2}\right) \\
\phi^{\prime}=\phi+\alpha^{\prime}\left[d_{6} R+d_{7} D^{2} \phi+d_{8}(\partial \phi)^{2}\right]+O\left(\alpha^{\prime 2}\right) .
\end{gathered}
$$

Since $G_{\mu \nu}$ and $\phi$ appear on an equal footing in the string effective action, it seems natural to consider the most general local covariant redefinitions that mix them. Note that the transformations of $G_{\mu \nu}$ that involve the dilaton do not have an interpretation as corresponding to a change of a renormalisation scheme in the standard $\sigma$-model $\beta$-functions. However, since the dilaton is a coupling constant of a $\sigma$-model defined on a curved $2 d$ background [10], such transformations should have a meaning of changes of a 'scheme' corresponding to the conformal anomaly coefficients $\bar{\beta}^{i}$.

The transformations $(8),(9)$ are to be understood within the $\alpha^{\prime}$ perturbation theory. Consider, for example, the simplest case of the linear dilaton background [1]

$$
G_{\mu \nu}=\eta_{\mu \nu}, \quad \phi=\phi_{0}+n_{\mu} x^{\mu}, \quad \frac{1}{6}(D-C)+\alpha^{\prime} n^{2}=0
$$

Making the redefinition (8), i.e.

$$
G_{\mu \nu}^{\prime}=\eta_{\mu \nu}+\alpha^{\prime} \partial_{\mu} \phi \partial_{\nu} \phi F_{1}\left(\alpha^{\prime} \partial \phi \partial \phi\right)+\eta_{\mu \nu} F_{2}\left(\alpha^{\prime} \partial \phi \partial \phi\right)=c_{1} \eta_{\mu \nu}+c_{2} \alpha^{\prime} n_{\mu} n_{\nu}
$$

one can significantly alter the structure of the metric (for example, change its signature or even make it degenerate) unless $\left|\alpha^{\prime} n^{2}\right| \ll 1$. Unless $C=D$ (i.e. unless $n^{2}=\eta_{\mu \nu} n^{\mu} n^{\nu}=0$ ) one cannot, strictly speaking, use the $\alpha^{\prime}$-perturbation theory since here the derivative of the dilaton (or dilaton 'momentum') is not small compared with $\alpha^{\prime-1 / 2}$. One can, however,

3 One can ignore the term $D_{\mu} D_{\nu} \phi$ in (8) since it can be eliminated by a coordinate transformation along $D^{\mu} \phi$. 
consider the linear dilaton background as a part of more general solution where $\alpha^{\prime} n^{2}$ is a free parameter.

With similar clarifications, we would like to suggest that the transformations $(8),(9)$ are perfectly admissible. The effective action $S$ and the $\bar{\beta}^{i}$-functions should have a background-independent meaning: the use of the flat space string $S$-matrix (or perturbation theory near any other particular background) is a technical tool for establishing the structure of $S$. Having determined it, one may relax all assumptions $(\operatorname{such} C=D)$ about a background used and consider all solutions of the same background-independent equations on an equal footing. Moreover, one is free to choose any representative in a class of equivalent actions (related by field redefinitions of the general type (8),(9)) and use it as a starting point. Different choices of the actions correspond to different off-shell extensions of string theory. Unless one has an extra principle for fixing a particular one, one is free to make arbitrary redefinitions like $(8),(9)$.

\section{Exact ' $S L(2, R) / U(1)$ ' solution and its relation to the leading-order $D=2$ black-hole background}

Let us now apply the above general remarks to the case of a particular $D=2$ solution of the bosonic string theory. Equations (6),(7) have the following leading-order (Euclidean 'black-hole') solution [12] 13]

$$
\begin{aligned}
& d s^{2}=d x^{2}+a^{2}(x) d \theta^{2}, \quad a^{2}(x)=\tanh ^{2} n x+O\left(\alpha^{\prime}\right), \\
& \phi=\phi_{0}-\ln \cosh n x+O\left(\alpha^{\prime}\right), \quad \frac{1}{6}(D-C)+\alpha^{\prime} n^{2}=0 .
\end{aligned}
$$

For this background

$$
R=\frac{4 n^{2}}{\cosh ^{2} n x}, \quad(\partial \phi)^{2}=n^{2} \tanh ^{2} n x, \quad-\frac{1}{2} D^{2} \phi+(\partial \phi)^{2}=\frac{1}{4} R+(\partial \phi)^{2}=4 n^{2}
$$


Since $\bar{\beta}^{\phi}$ in (7) is constant on a solution of $\bar{\beta}^{G}=0$ (6) [14, one may keep $n$ as a free parameter by not imposing the central-charge constraint (7). 6 Solving the two-loop metric equation in the standard scheme, where it has the form (6), one finds the following corrections $[15$

$$
\begin{gathered}
a^{2}(x)=\tanh ^{2} n x+2 \alpha^{\prime} n^{2} \tanh ^{4} n x+O\left(\alpha^{\prime 2}\right), \\
\phi=\phi_{0}-\ln \cosh n x+\frac{1}{2} \alpha^{\prime} n^{2} \tanh ^{2} n x+O\left(\alpha^{\prime 2}\right) .
\end{gathered}
$$

It was found that in the three- [15] and four- [16] loop approximation there exists such a scheme that the resulting solution reproduces the $\alpha^{\prime}$-expansion of the following generalisation of $(11),(12)$ [17]:

$$
\begin{gathered}
a^{2}(x)=\frac{\tanh ^{2} n x}{1-p \tanh ^{2} n x}=\frac{\left(1+2 \alpha^{\prime} n^{2}\right) \tanh ^{2} n x}{1+2 \alpha^{\prime} n^{2}(\cosh n x)^{-2}}, \quad p \equiv \frac{2 \alpha^{\prime} n^{2}}{1+2 \alpha^{\prime} n^{2}} \\
\phi=\phi_{0}-\ln \cosh n x-\frac{1}{4} \ln \left[1-p \tanh ^{2} n x\right] \\
=\phi_{0}^{\prime}-\ln \cosh n x-\frac{1}{4} \ln \left[1+2 \alpha^{\prime} n^{2}(\cosh n x)^{-2}\right] .
\end{gathered}
$$

This suggests that there exists a 'standard' scheme in which $(16),(17)$ is an exact solution, in agreement with the derivation of this background from the $S L(2, R) / U(1)$ coset conformal field theory [17] and the corresponding gauged WZNW model [18] [19] 6

The schemes for the $\beta$-functions considered in [15] [16] were related to the 'dimensional regularisation plus minimal subtraction' one by local redefinitions that did not include the

4 As was mentioned above, to avoid the question about the validity of perturbation theory in $\alpha^{\prime}$ once (7) is imposed, we may assume that this background is a part of a more general solution so that the total $C$ and $D$ are such that $\alpha^{\prime} n^{2}$ is effectively small. To make $\alpha^{\prime} n^{2}$ a continuous parameter we may introduce, for example, a linear dilaton background $\left(-n_{0} t\right)$ in the direction of an additional time-like coordinate so that $n^{2}-n_{0}^{2}=0$.

5 The two-loop contributions to the central-charge equation cancel out. In the standard scheme used in (6),(7), the total contribution to $\bar{\beta}^{\phi}$ comes from the asymptotic large-distance value of the 'one-loop' dilaton term $(\partial \phi)^{2}$.

6 If one imposes the condition that the total central charge $C=2+6 \alpha^{\prime} n^{2}$ in (7),(12) is equal to that of the $S L(2, R) / U(1)$ coset model, $C=3 k /(k-2)-1$, then the parameter $\alpha^{\prime} n^{2}$ becomes related to $k: \alpha^{\prime} n^{2}=1 /(k-2), p=2 / k$. 
dilaton-dependent terms. The result of [15] was that it is in such schemes that the leadingorder solution $(11),(12)$ is necessarily modified by the $\alpha^{\prime}$ corrections at higher loop orders (with the two-loop terms having the unambiguous form $(14),(15)$ ).

The main observation we would like to make here is that the exact background (16),(17) can be represented as a local dilaton-dependent field redefinition of the leadingorder one $(11),(12)$. Given that $(16),(17)$ is the exact solution in the 'standard' scheme, this implies that there exists a 'non-standard' scheme in which the leading-order solution $(11),(12)$ is, in fact, an exact solution to all orders in $\alpha^{\prime}$. In such a scheme each of the higher-loop contributions to $\bar{\beta}^{G}$ vanishes separately on the background (11),(12).

Consider the following field redefinition in $D=2$ (cf. (8),(9))

$$
\begin{gathered}
G_{\mu \nu}^{\prime}=G_{\mu \nu}+\alpha^{\prime} \partial_{\mu} \phi \partial_{\nu} \phi F_{1}\left(\alpha^{\prime} R, \alpha^{\prime} \partial \phi \partial \phi\right)+G_{\mu \nu} F_{2}\left(\alpha^{\prime} R, \alpha^{\prime} \partial \phi \partial \phi\right) \\
\phi^{\prime}=\phi+F_{3}\left(\alpha^{\prime} R, \alpha^{\prime} \partial \phi \partial \phi\right)
\end{gathered}
$$

where the functions $F_{s}$ are given by power series of their arguments. Taking

$$
F_{1}=-2+O\left(\alpha^{\prime}\right), \quad F_{2}=2 \alpha^{\prime}(\partial \phi)^{2}+O\left(\alpha^{\prime 2}\right), \quad F_{3}=-\frac{1}{8} \alpha^{\prime} R+O\left(\alpha^{2}\right)
$$

one finds that (18),(19) computed for (11),(12) reproduce the $\alpha^{\prime}$-corrections $(14),(15)$ to the leading-order solution. This means that making the redefinition $(18),(19),(20)$ in $(6),(7)$ one will find the two-loop equations corresponding to a 'scheme' in which the leading-order solution (11),(12) remains also the solution in the $\alpha^{\prime}$ - approximation.

The exact form of the redefinition that transforms (11),(12) into the $\alpha^{\prime}$-dependent background (16),(17) is given by (18),(19), with

$$
F_{1}=\frac{-2}{1+\frac{1}{2} \alpha^{\prime} R}, \quad F_{2}=\frac{2 \alpha^{\prime}(\partial \phi)^{2}}{1+\frac{1}{2} \alpha^{\prime} R}, \quad F_{3}=-\frac{1}{4} \ln \left(1+\frac{1}{2} \alpha^{\prime} R\right)
$$

7 It is interesting to note that the leading order correction term in (18),(21) $-2 \alpha^{\prime} \partial_{\mu} \phi \partial_{\nu} \phi$ is precisely the same that appears (along with the dilaton term) in the determinant produced by integration over the gauge field in the corresponding gauged WZNW action. This determinant was computed in [20]. The term $\sim \int \partial_{\mu} \phi \partial_{\nu} \phi \partial_{a} x^{\mu} \partial^{a} x^{\nu}$ in the resulting $2 d$ effective action can be ignored in the leading one-loop approximation but contributes at the two-loop level (there will be also other two-loop terms; the form of all such terms will depend on a computational scheme used). 
This is readily checked using (13). As a consequence, there exists a scheme (found by applying (18),(19),(21) to the 'standard' scheme) in which $(11),(12)$ is an exact solution. The main point is that the transformation between $(11),(12)$ and $(16),(17)$ can be represented in a local background-independent form. Note that the transformation for the inverse $D=2$ metric

$$
\begin{gathered}
G^{\mu \nu \prime}=G^{\mu \nu} f_{1}+\alpha^{\prime} D^{\mu} \phi D^{\nu} \phi f_{2} \\
f_{1}=\frac{1+\frac{1}{2} \alpha^{\prime} R}{1+\frac{1}{2} \alpha^{\prime}\left(R+4 \partial_{\mu} \phi \partial^{\mu} \phi\right)}, \quad f_{2}=\frac{2}{1+\frac{1}{2} \alpha^{\prime}\left(R+4 \partial_{\mu} \phi \partial^{\mu} \phi\right)},
\end{gathered}
$$

takes a simple form on the leading order solution (11),(12) (denominators in (22) become constant, cf. (13)) but still contains all higher order terms when represented in the background-independent form (22).

Since the transformation $(18),(19),(21)$ is invariant under constant shifts of $x$ (e.g. $\left.x \rightarrow x+\frac{1}{2} i \pi / n\right)$, it also transforms the dual to the leading-order solution (11),(12) (with cosh replaced by sinh) into the exact background 'dual' to (16),(17). As a consequence, in the 'non-standard' scheme in which (11),(12) is an exact solution, its dual in the sense of the usual leading-order form of duality

$$
\tilde{G}_{\theta \theta}=G_{\theta \theta}^{-1}, \quad \tilde{\phi}=\phi-\frac{1}{2} \ln G
$$

is also an exact solution. Therefore, the duality transformation also has its leading-order form in such a scheme. To find the exact $\left(\alpha^{\prime}\right.$-corrected [21]) form that the duality transformation has in the 'standard' scheme, one is to invert the transformation (18),(19),(21), then apply the leading-order duality (23), and finally transform the result again with the help of (18),(19),(21). The product of the three transformations will be given by a power series in $\alpha^{\prime}$ and will relate, in particular, (16),(17) to its exact dual (with $x \rightarrow x+\frac{1}{2} i \pi / n$ ). 


\section{Tachyon equation in a background}

One is left with the question about the physical implications of the existence of a scheme in which the leading-order background (11),(12) is an exact solution. While all higher string modes have zero condensates for this solution one should look at linearised equations for their perturbations in order to understand how the string feels the background (i.e. one should perturb the conformal theory by marginal operators as 'probes'). In general, these equations (or corresponding Weyl anomaly $\bar{\beta}$-functions) are linear differential equations with background field dependent coefficients which are also scheme dependent. It may happen that in the scheme where the background fields have semiclassical form the equations for the 'probes' still contain $\alpha$-corrections of all orders. This, in fact, what happens in the $D=2$ case considered above: while the equation for the tachyon has a simple form in the scheme where $(16),(17)$ is the solution, it becomes complicated in the new scheme. Being evaluated on the corresponding metric-dilaton solution, the tachyon equation takes of course the same $\alpha^{\prime}$-dependent form in both schemes (coinciding with the $\left(L_{0}+\bar{L}_{0}\right) T=2 T$ equation of conformal theory approach [17] $)$.

The tachyon $\bar{\beta}$-function has the following general structure [22][23] 24] (see also [25] [26])

$$
\bar{\beta}^{T}=-\gamma T+W^{\mu} \partial_{\mu} T-2 T,
$$

where $\gamma$ is the scalar anomalous dimension operator and $W^{\mu}$ is the 'diffeomorphism vector' [24]

$$
\begin{gathered}
\gamma=\Omega_{2}^{\mu \nu} D_{(\mu} D_{\nu)}+\sum_{n=3}^{\infty} \Omega_{n}^{\mu_{1} \ldots \mu_{n}} D_{\left(\mu_{1} \ldots D_{\left.\mu_{n}\right)}\right.}, \\
\Omega_{2}^{\mu \nu}=\frac{1}{2} \alpha^{\prime} G^{\mu \nu}+p_{1} \alpha^{\prime 2} R^{\mu \nu}+p_{2} \alpha^{\prime 2} H^{\mu \alpha \beta} H_{\alpha \beta}^{\nu}+p_{3} \alpha^{\prime 2} R_{\alpha \beta \gamma}^{\mu} R^{\nu \alpha \beta \gamma}+\ldots, \\
\Omega_{3}^{\mu \nu \rho}=\alpha^{\prime 4} q_{1} D_{\alpha} R_{\beta}^{\mu \nu}{ }_{\gamma} R^{\alpha \beta \gamma \rho}+\ldots, \quad \Omega_{4}^{\mu \nu \rho \lambda}=\alpha^{\prime 4} s_{1} R^{\mu \alpha \beta \nu} R_{\alpha \beta}^{\rho}{ }^{\lambda}+\ldots,
\end{gathered}
$$

8 Here we consider the case of arbitrary dimension $D$ and include also the dependence on the field strength $H$ of a possible antisymmetric tensor background. 


$$
\begin{gathered}
W_{\mu}=\alpha^{\prime} \partial_{\mu} \phi+M_{\mu}(G, H) \\
M_{\mu}=t_{1} \alpha^{\prime 2} \partial_{\mu}\left(H_{\alpha \beta \gamma}^{2}\right)+t_{2} \alpha^{\prime 2} D^{\nu}\left(H_{\mu \alpha \beta} H_{\nu}{ }^{\alpha \beta}\right)+t_{3} \alpha^{\prime 3} \partial_{\mu}\left(R_{\alpha \beta \gamma \rho}^{2}\right)+\ldots .
\end{gathered}
$$

The coefficients $p_{1}, p_{2}, \ldots$ are obviously scheme-dependent. In the dimensional regularisation / minimal subtraction scheme $p_{1}=0$ [23] [24], the coefficients $p_{2}, t_{1}, t_{2}$ can be inferred from [7][26], $p_{3}=\frac{3}{16}, t_{3}=\frac{1}{32}$ [27] and the four-loop coefficients in $\Omega_{2}$ and $q_{1}, s_{1}, \ldots$ were found in [28]. Thus if $H=0$ the leading order (one-loop) form of the tachyon equation

$$
\bar{\beta}^{T}=-\frac{1}{2} \alpha^{\prime} D^{2} T+\alpha^{\prime} D^{\mu} \phi \partial_{\mu} T-2 T=0,
$$

is not modified by the two-loop corrections in this scheme.

Let us now recall that the exact $D=2$ background (16),(17) was found in [17] by identifying the $\left(L_{0}+\bar{L}_{0}\right) T=2 T$ equation of $S L(2, R) / U(1)$ coset conformal theory with the leading order form of the tachyon equation (29). The consistency of such identification then demands that the scheme in which $(16),(17)$ is the exact solution of the metric-dilaton $\bar{\beta}^{i}=0$ equations (which exists according to [15] [16] in the four-loop approximation) must be the one in which the tachyon $\bar{\beta}$-function maintains its leading-order form (29). This, in fact, is easy to check in the three loop approximation: the $R^{2}$-term appearing in (26) in the minimal subtraction scheme [27] is redefined away by the same transformation (from the minimal subtraction scheme to the 'standard' one corresponding to $(16),(17))$ that was found in 15$] .9$ We expect that similar statement is true in four (and higher) loop approximation (to get rid of higher derivative terms in (25) one will need also to redefine the tachyon field). As a result, one can define the scheme in which (16),(17) is an exact solution as the one in which $\bar{\beta}^{T}$ takes the simple form (29).

In conclusion, let us emphasize that the existence of a local, covariant and backgroundindependent transformation, which, like (18),(19),(21), relates the leading-order solution to

9 Let us note that the expressions for the coefficients $a_{i}$ in terms of $c_{i}$ in eq.(23) of ref. 15] had erroneously extra factors of 2 . 
the exact one, is quite non-trivial (as we have noted above, a generic exact solution is nonlocal, being expressed (2) in terms of the leading-order one). Equivalently, the existence of a 'scheme' in which a leading-order solution is exact to all orders should be a property of only a very special class of solutions. Given that the group spaces and now the simplest $S L(2, R) / U(1)$ coset model background belong to such a class, one may conjecture that this is actually true for all solutions that correspond to $G / H$ coset models, i.e. for all $\sigma$-model backgrounds that originate from gauged WZNW theories.

However, this fact may have rather limited importance since the quantum string modes propagating in these backgrounds will always 'feel' the $\alpha^{\prime}$-corrected solutions: the corresponding equations will be equivalent to the $\left(L_{0}+\bar{L}_{0}\right) T=n T$ - equations of the coset $G / H$ conformal field theories which depend non-trivially on the level $k$ in all (bosonic) cases with the subgroup $H \neq 1$. This suggests that the 'standard' scheme in which the tachyon equation has the simple form (29) and the background fields contain $1 / k$ corrections of all orders is a 'preferrred' one being directly related to the conformal field theory interpretation.

\section{Acknowledgements}

I am grateful to K. Sfetsos for a collaboration on related issues [29] and to Al.B. Zamolodchikov for a stimulating discussion. I would like to thank H. Osborn for raising the important question about the form of the tachyon $\beta$-function and I. Jack and J. Russo for comments. I acknowledge also a support of SERC. 


\section{References}

[1] J. Scherk and J. Schwarz, Nucl. Phys. B81(1974)118.

[2] A.A. Tseytlin, Phys. Lett. B176(1986)92; Nucl. Phys. B276(1986)391.

[3] D. Gross and E. Witten, Nucl. Phys. B277(1986)1.

[4] T.L. Curtright and C.K. Zachos, Phys. Rev. Lett. 53(1984)1799; S. Mukhi, Phys. Lett. B162(1985)345; S. de Alwis, Phys. Lett. B164(1985)67.

[5] E. Witten, Commun. Math. Phys. 92(1984)455.

[6] C. Hull and P.K. Townsend, Phys. Lett. B191(1987)115; D. Zanon, Phys. Lett. B191(1987)363; D.R.T. Jones, Phys. Lett. B191(1987)363; S. Ketov, Nucl. Phys. B294(1987)813.

[7] R.R. Metsaev and A.A. Tseytlin, Phys.Lett. B191(1987)354; Nucl.Phys. B293(1987)385.

[8] D. Nemeschansky and S. Yankielowicz, Phys.Rev.Lett. 54(1985)620; 54(1985)1736(E).

[9] C.G. Callan, D. Friedan, E. Martinec and M.J. Perry, Nucl. Phys.B262(1985)593.

[10] E.S. Fradkin and A.A. Tseytlin, Phys.Lett. B158(1985)316; Nucl.Phys. B261(1985)1.

[11] R. Myers, Phys.Lett. B199(1987)371; I. Antoniadis, C. Bachas, J. Ellis and D. Nanopoulos, Phys. Lett. B211(1988)393.

[12] S. Elitzur, A. Forge and E. Rabinovici, Nucl. Phys. B359 (1991) 581; G. Mandal, A. Sengupta and S. Wadia, Mod. Phys. Lett. A6(1991)1685.

[13] E. Witten, Phys. Rev. D44(1991)314.

[14] G. Curci and G. Paffuti, Nucl. Phys. B286(1987)399.

[15] A.A. Tseytlin, Phys. Lett. B268(1991)175.

[16] I. Jack, D.R.T. Jones and J. Panvel, Nucl. Phys. B393(1993)95.

[17] R. Dijkgraaf, H. Verlinde and E. Verlinde, Nucl. Phys. B371(1992)269.

[18] A.A. Tseytlin, Nucl. Phys. B399(1993)601.

[19] I. Bars and K. Sfetsos, Phys. Rev. D48(1993)844.

[20] A.S. Schwarz and A.A. Tseytlin, Nucl. Phys. B399(1993)691.

[21] A.A. Tseytlin, Mod. Phys. Lett. A6(1991)1721.

[22] D.H. Friedan, Phys. Rev. Lett. 45(1980)1057; Ann. Phys. (NY) 163(1985)318.

[23] C.G. Callan and Z. Gan, Nucl. Phys. B272(1986)647.

[24] A.A. Tseytlin, Phys. Lett. B178(1986)34.

[25] A.A. Tseytlin, Int. J. Mod. Phys. A4(1989)1257.

[26] H. Osborn, Ann. Phys. (NY) 200(1990)1.

[27] I. Jack, D.R.T. Jones and D.A. Ross, Nucl. Phys. B307(1988)130.

[28] I. Jack, D.R.T. Jones and N. Mohammedi, Nucl. Phys. B332(1990)333.

[29] K. Sfetsos and A.A. Tseytlin, preprint CERN-TH.6969/93. 